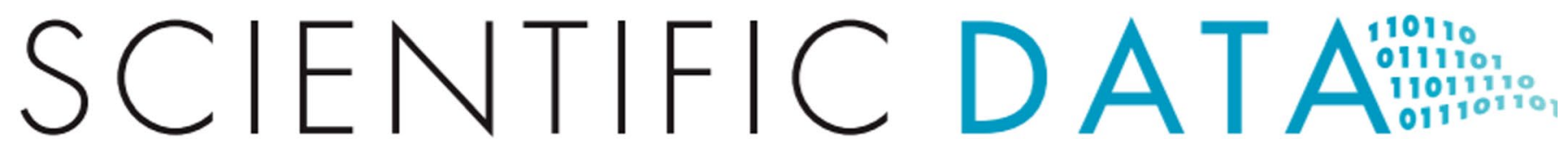

Check for updates

OPEN

COMMENT

\section{COVID-19 Disease Map, building a computational repository of SARS-CoV-2 virus-host interaction mechanisms}

\author{
Marek Ostaszewski $\mathbb{D}^{1}$, Alexander Mazein $\mathbb{D D}^{1,2}$, Marc E. Gillespie $\mathbb{D}^{3,4}$, Inna Kuperstein ${ }^{5}$, \\ Anna Niarakis $\mathbb{D}^{6}$, Henning Hermjakob $\mathbb{D}^{7}$, Alexander R. Pico ${ }^{8}$, Egon L. Willighagen $\mathbb{D}^{9}$, \\ Chris T. Evelo ${ }^{9,10}$, Jan Hasenauer ${ }^{11,12,13}$, Falk Schreiber ${ }^{14,15}$, Andreas Dräger ${ }^{16,17,18}$, \\ Emek Demir $^{19}$, Olaf Wolkenhauer ${ }^{20,21}$, Laura I. Furlong ${ }^{22}$, Emmanuel Barillot ${ }^{5}$, \\ Joaquin Dopazo ${ }^{23,24,25,26}$, Aurelio Orta-Resendiz ${ }^{27,28}$, Francesco Messina ${ }^{29,30}$, \\ Alfonso Valencia ${ }^{31,32}$, Akira Funahashi $\mathbb{D}^{33}$, Hiroaki Kitano ${ }^{34,35,36}$, Charles Auffray ${ }^{2}$, \\ Rudi Balling ${ }^{1} \&$ Reinhard Schneider $\mathbb{D}^{1 \times}$
}

Researchers around the world join forces to reconstruct the molecular processes of the virushost interactions aiming to combat the cause of the ongoing pandemic. 


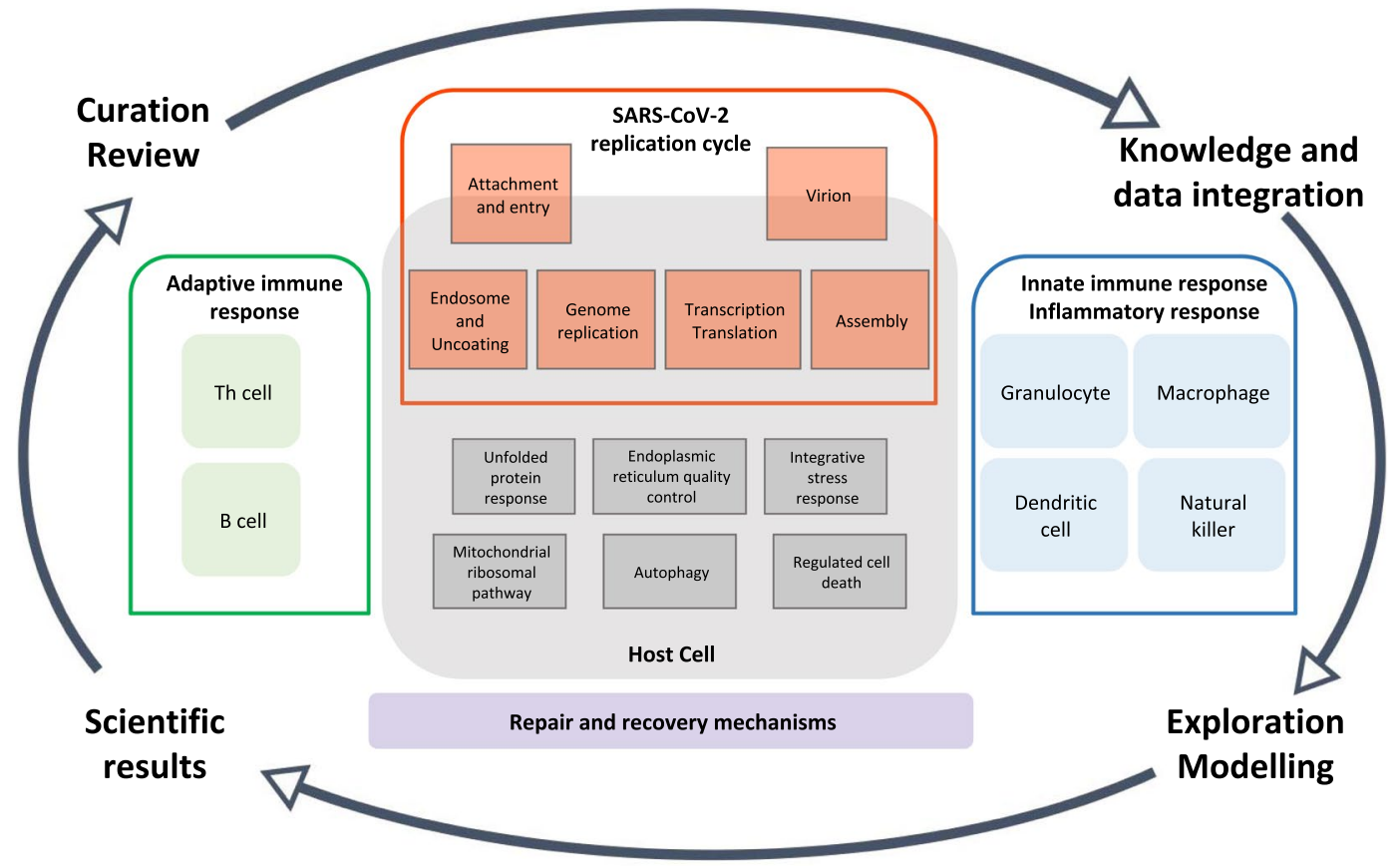

Fig. 1 The overview of the COVID-19 Disease Map project. The map focuses on SARS-CoV-2 replication cycle, its interactions with the host, reaction of the immune system and repair mechanisms. The curated and reviewed content will be continuously integrated and cross-linked with data and knowledge bases, to support visual and computational exploration, as well as disease modelling efforts. The acquired results will benefit the research community and provide feedback to refine the scope of curation activities.

W

e announce the COVID-19 Disease Map (https://doi.org/10.17881/covid19-disease-map), an effort to build a comprehensive, standardized knowledge repository of SARS-CoV-2 virus-host interaction mechanisms, guided by input from domain experts and based on published work. This knowledge, available in the vast body of existing literature ${ }^{1,2}$ and the fast-growing number of new SARS-CoV-2 publications, needs rigorous and efficient organization in both human and machine-readable formats.

This endeavour is an open collaboration between clinical researchers, life scientists, pathway curators, computational biologists and data scientists. Currently, 162 contributors from 25 countries around the world are participating in the project, including partners from Reactome ${ }^{3}$, WikiPathways ${ }^{4}$, IMEx Consortium ${ }^{5}$, Pathway Commons $^{6}$, DisGeNET ${ }^{7}$, ELIXIR $^{8}$, and the Disease Maps Community ${ }^{9}$ With this effort, we aim for long-term community-based development of high-quality models and knowledge bases, linked to data repositories.

The COVID-19 Disease Map will be a platform for visual exploration and computational analyses of molecular processes involved in SARS-CoV-2 entry, replication, and host-pathogen interactions, as well as immune response, host cell recovery and repair mechanisms. The map will support the research community and improve our understanding of this disease to facilitate the development of efficient diagnostics and therapies. Figure 1 illustrates the initial scope and layout of the map and its life cycle.

At the time this Comment went to press, the COVID-19 Disease Map contains pathways of (i) the virus replication cycle and its transcription mechanisms; (ii) SARS-CoV-2 impact on ACE2-regulated pulmonary blood pressure, apoptosis, Cul2-mediated ubiquitination, heme catabolism, Interferon 2 and PAMP signalling, and endoplasmic reticulum stress; (iii) SARS-CoV-2 proteins Nsp4, Nsp6, Nsp14 and Orf3a. Moreover, the map incorporates the COVID-19 collection of WikiPathway diagrams ${ }^{10}$ and a pre-published genome-scale metabolic model of human alveolar macrophages with SARS-CoV-2 ${ }^{11}$. All these contributed open-access resources are referenced at https://fairdomhub.org/projects/190\#models.

By combining diagrammatic representation of COVID-19 mechanisms with underlying models, the map fulfils a dual role. First, it is a graphical, interactive representation of disease-relevant molecular mechanisms linking different knowledge bases. Second, it is a computational resource of reviewed content for graph-based analyses $^{12}$ and disease modelling ${ }^{13}$. Thus, it provides a platform for domain experts, such as clinicians, virologists, and immunologists, to collaborate with data scientists and computational biologists for a rigorous model building, accurate data interpretation and drug repositioning. It offers a shared mental map to understand gender, age, and other susceptibility features of the host, disease progression, defence mechanisms, and response to treatment. Finally, it can be used together with the maps of other human diseases to study comorbidities.

In the construction of the COVID-19 Disease Map, we rely on multiple tools for curation and review the contributed content in a distributed, on-the-fly manner. Most importantly, already at this early stage, we involve practising physicians and clinical researchers to improve the scope and quality of the map. Motivated by our curation experience and the number of participants contributing to the construction of the map, we propose and regularly revise common curation guidelines and follow commonly-accepted exchange standards. Moreover, given 
the multicellular and multiorgan nature of COVID-19 infection and the complexity of the underlying molecular mechanisms, we envisage the map as a hierarchical structure of interconnected functional modules. We anticipate that the structure of the map will evolve as new knowledge about the disease is revealed.

This distributed, multi-tool, multi-group approach is dictated by the urgency of the ongoing pandemic, by the high volume of new COVID-19-related publications, and by an impressive response from the research community. In this challenging situation, it is imperative that community-based approaches are used to develop high-quality models and data. To ensure a transparent view of the contributors and community resources, we rely on the support of FAIRDOMHub ${ }^{14}$. All data and curation guidelines related to the COVID-19 Disease Map are available at https://fairdomhub.org/projects/190.

We invite curators to join the project and contribute to building a solid foundation of COVID-19 molecular and cellular mechanisms using systems biology standards ${ }^{15-17}$. Moreover, we request support from domain experts to advise on the content and to review the map, improving its quality and applicability, as well as experts in modelling to accelerate the development of efficient diagnoses, treatments, and vaccines in response to the ongoing pandemic.

Received: 14 April 2020; Accepted: 24 April 2020;

Published online: 05 May 2020

\section{References}

1. Fung, T. S. \& Liu, D. X. Human Coronavirus: Host-Pathogen Interaction. Annu. Rev. Microbiol. 73, 529-557 (2019).

2. COVID-19 Open Research Dataset (CORD-19). Semantic Scholar https://pages.semanticscholar.org/coronavirus-research (2020).

3. Jassal, B. et al. The Reactome pathway knowledgebase. Nucleic Acids Res 48, D498-D503 (2020).

4. Slenter, D. N. et al. WikiPathways: a multifaceted pathway database bridging metabolomics to other omics research. Nucleic Acids Res 46, D661-D667 (2018).

5. Orchard, S. et al. Protein interaction data curation: the International Molecular Exchange (IMEx) consortium. Nat. Methods 9 , 345-350 (2012).

6. Rodchenkov, I. et al. Pathway Commons 2019 Update: integration, analysis and exploration of pathway data. Nucleic Acids Res 48 , D489-D497 (2020).

7. Piñero, J. et al. The DisGeNET knowledge platform for disease genomics: 2019 update. Nucleic Acids Res 48, D845-D855 (2020).

8. Drysdale, R. et al. The ELIXIR Core Data Resources: fundamental infrastructure for the life sciences. Bioinforma. Oxf. Engl., https:// doi.org/10.1093/bioinformatics/btz959 (2020).

9. Mazein, A. et al. Systems medicine disease maps: community-driven comprehensive representation of disease mechanisms. NPJ Syst. Biol. Appl 4, 21 (2018).

10. COVID-19 Pathway Collection. WikiPathways https://www.wikipathways.org/index.php/Portal:Disease/COVIDPathways (2020).

11. Renz, A., Widerspick, L. \& Dräger, A. FBA reveals guanylate kinase as a potential target for antiviral therapies against SARS-CoV-2 (Version 3). Preprint at https://doi.org/10.5281/ZENODO.3752641 (2020).

12. Villaveces, J. M., Koti, P. \& Habermann, B. H. Tools for visualization and analysis of molecular networks, pathways, and -omics data. Adv. Appl. Bioinforma. Chem. AABC 8, 11-22 (2015).

13. Ostaszewski, M. et al. Community-driven roadmap for integrated disease maps. Brief. Bioinform 20, 659-670 (2019).

14. Wolstencroft, K. et al. FAIRDOMHub: a repository and collaboration environment for sharing systems biology research. Nucleic Acids Res 45, D404-D407 (2017).

15. Le Novère, N. et al. The Systems Biology Graphical Notation. Nat. Biotechnol. 27, 735-741 (2009).

16. Hucka, M. et al. The Systems Biology Markup Language (SBML): Language Specification for Level 3 Version 2 Core Release 2. J. Integr. Bioinforma. 16,1-181 (2019).

17. Demir, E. et al. The BioPAX community standard for pathway data sharing. Nat. Biotechnol. 28, 935-942 (2010).

\section{Acknowledgements}

We would like to acknowledge the members of the COVID-19 Disease Map Community. Their support, contributions and engagement make this project possible. All the members of the COVID-19 Disease Map Community can be found at https://fairdomhub.org/projects/190. We would also like to acknowledge the FAIRDOMHub project members, in particular Martin Golebiewski, Carole Goble, Stuart Owen and Xiaoming $\mathrm{Hu}$, for their support for COVID-19 Disease Map project on the FAIRDOMHub platform (https://fairdomhub. org/). The Responsible and Reproducible Research (R3) team of the Luxembourg Centre for Systems Biomedicine is acknowledged for supporting the project and promoting reproducible research.

\section{Author contributions}

All the authors have written and revised the manuscript.

\section{Competing interests}

$\mathrm{RB}$ is a founder and a shareholder of MEGENO S.A., and a shareholder of ITTM S.A. RS is a founder and a shareholder of MEGENO S.A. and ITTM S.A. Remaining authors declare no competing interests.

\section{Additional information}

Correspondence and requests for materials should be addressed to R.S.

Reprints and permissions information is available at www.nature.com/reprints.

Publisher's note Springer Nature remains neutral with regard to jurisdictional claims in published maps and institutional affiliations. 
(1) Open Access This article is licensed under a Creative Commons Attribution 4.0 International License, which permits use, sharing, adaptation, distribution and reproduction in any medium or format, as long as you give appropriate credit to the original author(s) and the source, provide a link to the Creative Commons license, and indicate if changes were made. The images or other third party material in this article are included in the article's Creative Commons license, unless indicated otherwise in a credit line to the material. If material is not included in the article's Creative Commons license and your intended use is not permitted by statutory regulation or exceeds the permitted use, you will need to obtain permission directly from the copyright holder. To view a copy of this license, visit http://creativecommons.org/licenses/by/4.0/.

(C) The Author(s) 2020 